\title{
Evaluation of the individual $50 \%$ inhibitory area under the concentration curve of 5-fluorouracil based on the collagen gel droplet embedded culture drug sensitivity test in colorectal cancer
}

\author{
TAKUMI OCHIAI $^{1}$, KAZUHIKO NISHIMURA ${ }^{1}$, TOMOO WATANABE ${ }^{1}$, MASAYUKI KITAJIMA ${ }^{1}$, \\ MASATAKA TSUJI ${ }^{1}$, AKINORI NAKATANI ${ }^{1}$, NOBORU NAKAYAMA ${ }^{1}$, TAKASHI MARUSASA ${ }^{1}$, \\ SATOMI MASHIKO ${ }^{2}$, ISAO NAGAOKA ${ }^{3}$ and SHUNJI FUTAGAWA ${ }^{1}$ \\ Departments of ${ }^{1}$ Surgery, and ${ }^{2}$ Pharmacy, Tobu Chiiki Hospital, Tokyo Metropolitan Health and \\ Medical Treatment Corporation; ${ }^{3}$ Department of Host Defense and Biochemical Research, \\ Juntendo University School of Medicine, Tokyo, Japan
}

Received August 12, 2008; Accepted December 8, 2008

DOI: 10.3892/mmr_00000113

\begin{abstract}
We have previously reported the 5-fluorouracil (5-FU) sensitivity of cancer cells from colorectal cancer (CRC) patients using the collagen gel droplet embedded culture-drug sensitivity test (CD-DST) under multiple drug concentrations and contact durations. Moreover, the area under the concentration curve (AUC) and growth inhibition rate (IR) were combined, resulting in the AUC-IR curve, which was approximated to the logarithmic curve. In the present study, we used the AUC-IR curve to calculate the individualized $\mathrm{AUC}_{\mathrm{IR} 50}$, the AUC value that imparts $50 \%$ growth inhibition. Individual $\mathrm{AUC}_{\mathrm{IR} 50}$ was calculated in CRC patients, and its distribution was evaluated. The cumulative distribution of individual $\mathrm{AUC}_{\mathrm{IR} 50}$ was regressed over two lines (logarithmic scale). Among the 45 resectable CRC patients, those who achieved more than the individual $\mathrm{AUC}_{\mathrm{IR} 50}$ during post-operative 5-FUbased chemotherapy demonstrated a trend towards better disease-free survival compared to those who did not achieve $\mathrm{AUC}_{\mathrm{IR} 50}$. Of the Dukes' D patients $(\mathrm{n}=10)$, those who achieved more than twice the individual $\mathrm{AUC}_{\mathrm{IR} 50}$ during post-operative 5-FU-based chemotherapy demonstrated significantly better survival rates $(p=0.05)$ than those who did not. In this study, the distribution of the individual $\mathrm{AUC}_{\mathrm{IR} 50}$ suggested that approximately $6 \%$ of patients demonstrated very low 5 -FU sensitivity. Therefore, the individual $\mathrm{AUC}_{\mathrm{IR} 50}$ was useful in classifying good, intermediate and poor 5-FU response. Achievement of the individual $\mathrm{AUC}_{\mathrm{IR} 50}$ may be a prerequisite
\end{abstract}

Correspondence to: Dr Takumi Ochiai, Department of Surgery, Tobu Chiiki Hospital, Tokyo Metropolitan Health and Medical Treatment Corporation, 5-14-1 Kameari, Katsushika City, Tokyo 125-8512, Japan

E-mail: takumi-o@ma.kitanet.ne.jp

Key words: colorectal cancer, 5-fluorouracil, collagen gel droplet embedded drug sensitivity test, area under the concentration curve, individual $\mathrm{AUC}_{\mathrm{IR} 50}$ for individualized 5-FU-based adjuvant chemotherapy. As well, the early achievement of twice the individual $\mathrm{AUC}_{\text {IR50 }}$ may indicate an improved prognosis in Dukes' D patients. The individual $\mathrm{AUC}_{\mathrm{IR} 50}$ using CD-DST is useful in determining the individualized chemotherapy of CRC patients, thus CD-DST has the potential to facilitate the establishment of individualized chemotherapy for CRC.

\section{Introduction}

Individualized chemotherapy is a therapeutic strategy based on the features of a respective tumor, and has been proposed to improve patient clinical outcome while maintaining quality of life. In the field of gastrointestinal cancer, 5-fluorouracil (5-FU) is the drug most often used to assess antitumor effect. The drug sensitivity of tumor cells is one of the key factors that must be assessed in individualized chemotherapy for cancer patients. We have reported the 5-FU sensitivity of cancer cells from colorectal cancer (CRC) patients using the collagen gel droplet embedded culture-drug sensitivity test (CD-DST) under multiple drug concentrations and contact durations. Moreover, the area under the concentration curve (AUC) and growth inhibition rate (IR) were combined, resulting in the AUC-IR curve, which was approximated to the logarithmic curve (1). We have also reported that the growth inhibition rate, calculated from the AUC-IR curve, and the actual growth inhibition rate, at an AUC of $48 \mu \mathrm{g} \mathrm{x} \mathrm{h} / \mathrm{ml}$, are identical. Based on these results, we proposed that the in vitro antitumor effect of 5-FU depends on AUC in colorectal cancer, and that the AUC-IR curve is reliable (2). However, it remained to be elucidated whether the individual $\mathrm{AUC}_{\mathrm{IR} 50}$ the AUC value that imparts $50 \%$ growth inhibition with an AUC-IR curve - reflects clinical outcome.

This study aimed to calculate the individual $\mathrm{AUC}_{\text {IR50 }}$ using the AUC-IR curve, and to evaluate the distribution of the individual $\mathrm{AUC}_{\mathrm{IR} 50}$. The relationship between 5-FU sensitivity as determined by the AUC-IR curve and clinical outcome was also evaluated in early and advanced CRC patients treated with 5-FU-based chemotherapy. 


\section{Materials and methods}

Patients. Surgical specimens of the primary tumor were obtained from 63 colorectal cancer patients resected without pre-operative chemotherapy between January 2002 and September 2007. Informed consent for measuring drug sensitivity was obtained from all patients.

Methods. Tumor tissue was excised from the primary surgical specimen and applied to the CD-DST. The specimen was washed out five times with saline $(50 \mathrm{ml})$, then five times with antibiotic fluid $(50 \mathrm{ml})$ containing $1.0 \mathrm{mg} / \mathrm{ml}$ piperacillin and $0.5 \mathrm{mg} / \mathrm{ml} \mathrm{kanamycine.} \mathrm{The} \mathrm{transport} \mathrm{bottle} \mathrm{contained}$ $1.0 \mathrm{mg} / \mathrm{ml}$ piperacillin, $0.5 \mathrm{mg} / \mathrm{ml}$ kanamycine and $2.5 \mu \mathrm{g} / \mathrm{ml}$ amphotericin B. 5-FU tumor sensitivity was evaluated by CD-DST, performed as described by Kobayashi et al (3).

One gram of tissue was treated with dispersion enzyme cocktail containing $1.0 \%$ collagenase for $2 \mathrm{~h}$. Dispersed cell suspensions were inoculated in pre-culture media on collagencoated flasks overnight, and then viable tumor cells were recovered by $0.05 \%$ collagenase treatment. Recovered cells were embedded in 30- $\mu \mathrm{l}$ collagen gel droplets. Embedded cells were cultivated in culture media containing 5-FU at $0.2 \mu \mathrm{g} / \mathrm{ml}$ for $3 \mathrm{~h}, 1.0 \mu \mathrm{g} / \mathrm{ml}$ for $3 \mathrm{~h}, 10.0 \mu \mathrm{g} / \mathrm{ml}$ for $3 \mathrm{~h}, 0.2 \mu \mathrm{g} / \mathrm{ml}$ for $24 \mathrm{~h}, 1.0 \mu \mathrm{g} / \mathrm{ml}$ for $24 \mathrm{~h}, 10.0 \mu \mathrm{g} / \mathrm{ml}$ for $24 \mathrm{~h}, 0.2 \mu \mathrm{g} / \mathrm{ml}$ for $120 \mathrm{~h}, 1.0 \mu \mathrm{g} / \mathrm{ml}$ for $120 \mathrm{~h}$ and $10.0 \mu \mathrm{g} / \mathrm{ml}$ for $120 \mathrm{~h}$. After the removal of 5-FU-containing media, the cells were further cultured for 7 days in serum-free culture media to prevent the growth of fibroblasts. Viable cells were stained by neutral red solution and counted by the imaging colorimetric quantification method. The surviving cell number ratio between the drugtreated and control groups was calculated. Patients who recorded $\geq 4$ conditions as well as those with a growth rate $>0.8$ were regarded as successful cases.

After converting the drug concentrations and contact time to AUC, an AUC-IR curve was plotted against the growth inhibition rate, and the individual $\mathrm{AUC}_{\mathrm{IR} 50}$ - the $\mathrm{AUC}$ value that imparts $50 \%$ growth inhibition - was calculated from the AUC-IR regression curve. Subsequently, the distribution of the individual $\mathrm{AUC}_{\mathrm{IR} 50}$ and its correlation to patient background (histological type and Dukes' stage) were evaluated.

The 45 resectable CRC patients with Dukes' stages A, B or $\mathrm{C}$ who received 5-FU-based adjuvant chemotherapy were divided into achieved and non-achieved groups. The total administered AUC of 5-FU was more than the individual $\mathrm{AUC}_{\mathrm{IR} 50}$ in the achieved group, but less than the individual $\mathrm{AUC}_{\mathrm{IR} 50}$ in the non-achieved group. The disease-free survival (DFS) rates were evaluated and compared in the two groups.

The 10 Dukes' D patients who had distant metastasis and received post-operative 5-FU-based chemotherapy after the primary tumor was resected were also divided into achieved and non-achieved groups. The total administered AUC was more than twice the individual $\mathrm{AUC}_{\mathrm{IR} 50}$ in the achieved group, but less than twice the individual $\mathrm{AUC}_{\mathrm{IR} 50}$ in the non-achieved group. Overall survival (OS) rates were evaluated and compared in the two groups.

Statistics. The primary study endpoint was the OS, estimated as the time from the date of surgery to last contact or to the date of the patient succumbing to other causes. The secondary
Table I. Backgrounds of the 63 patients.

No. of patients

Mean age, years (range)

Gender (male/female)

Histological type (well/mod/poor/muc)

$8 / 48 / 2 / 5$

Dukes' stage $(\mathrm{A} / \mathrm{B} / \mathrm{C} / \mathrm{D})$

$5 / 30 / 16 / 12$

Colon/rectum

$51 / 12$

Well, well-differentiated; mod, moderately-differentiated; poor, poorly-differentiated; muc, mucinous carcinoma.

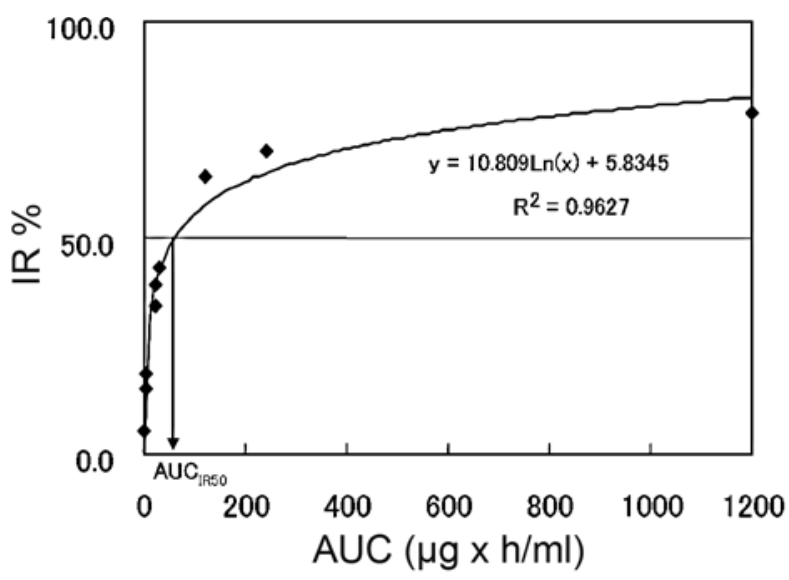

Figure 1. Growth inhibition according to a representative 5-fluorouracil area under the curve (AUC); IR, inhibition rate; $\mathrm{AUC}_{\mathrm{IR} 50}$, $\mathrm{AUC}$ value imparting $50 \%$ growth inhibition.

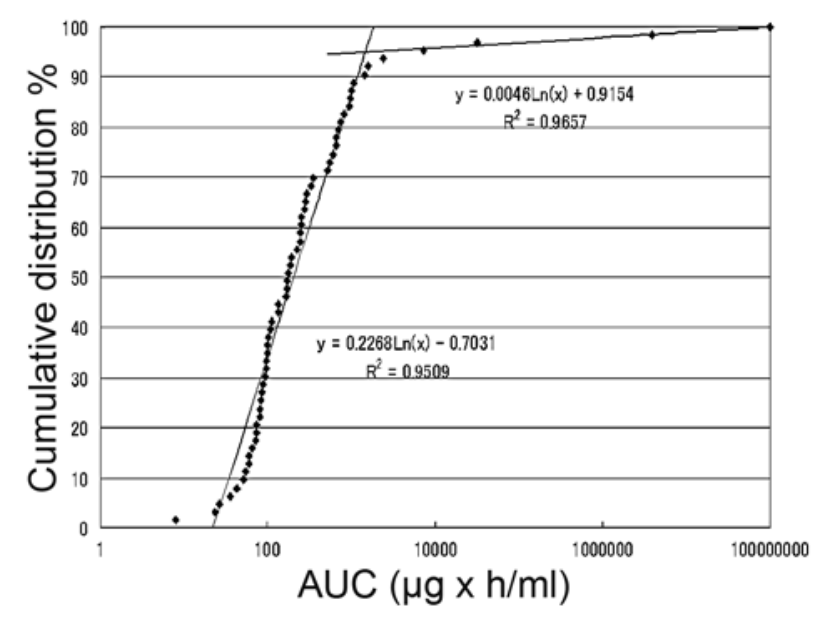

Figure 2. Cumulative distribution of individual $\mathrm{AUC}_{\mathrm{IR} 50}$.

endpoint was the DFS, estimated as the time from the date of surgery to the occurrence of any local or distant relapse or to the date of the patient succumbing to other causes.

Comparisons of patient characteristics in the 'achieved' and 'non-achieved' groups were compiled using the $\chi^{2}$ test, and gender and tumor site by the t-test. Age, Dukes' stage and histological type were estimated using Tukey's test. 


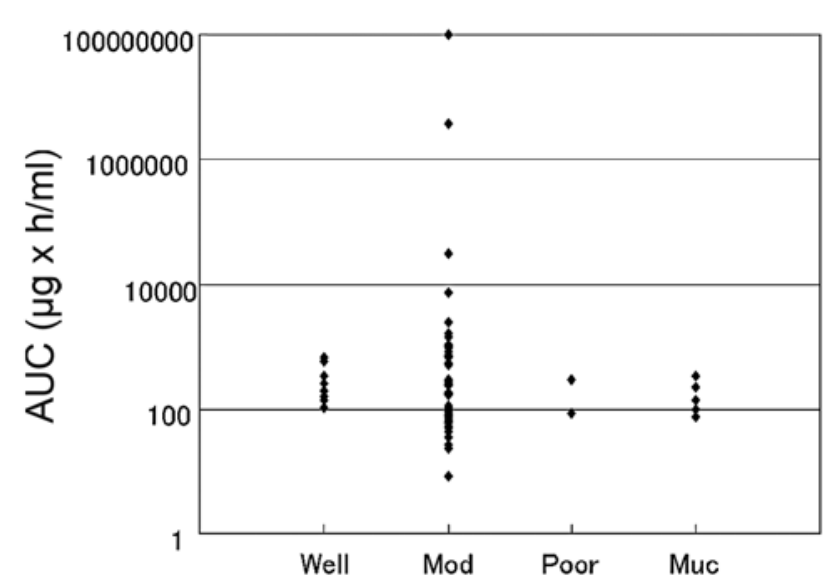

Figure 3. Distribution of individual $\mathrm{AUC}_{\mathrm{IR} 50}$ by histological type. Well, welldifferentiated; mod, moderately-differentiated; poor, poorly-differentiated; muc, mucinous carcinoma.

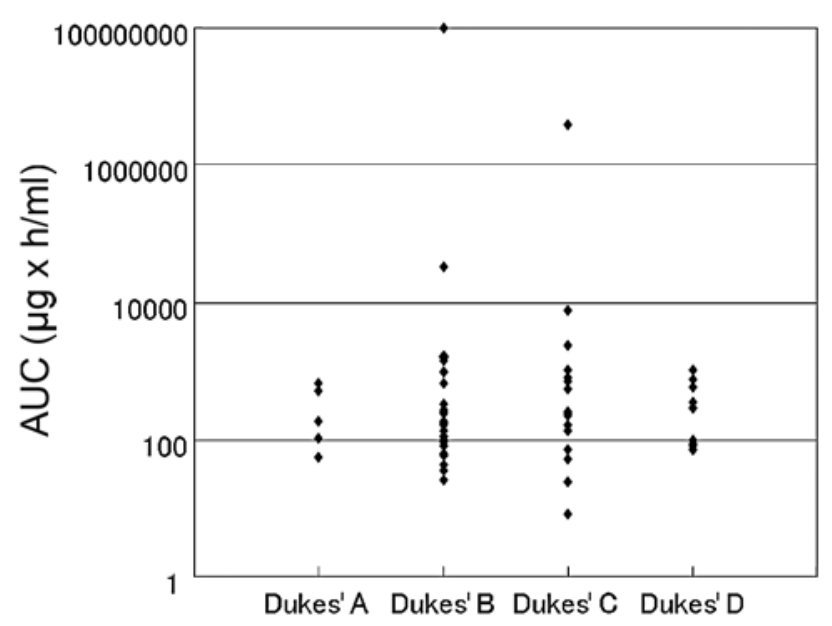

Figure 4. Distribution of individual $\mathrm{AUC}_{\mathrm{IR} 50}$ according to Dukes' stage.

OS and DFS were calculated using Kaplan-Meier analysis, and a primary and secondary comparison between the two groups was carried out using the log-rank test. Statistical tests were two-sided $(\alpha=0.05)$ and were carried out using the SPSS package (version II for Windows).

\section{Results}

The backgrounds of the 63 patients are shown in Table I. The AUC-IR curve of a representative patient is shown in Fig. 1. The approximate expression and correlation coefficients were $\mathrm{y}=10.809 \mathrm{Ln}(\mathrm{x})+5.8345\left(\mathrm{R}^{2}=0.9657\right)$ for $\mathrm{AUC}$, and $59.5 \mu \mathrm{g} \mathrm{x} \mathrm{h} / \mathrm{ml}\left[\mathrm{y}=10.809 \mathrm{Ln}(\mathrm{x})+5.8345, \mathrm{R}^{2}=0.963\right]$ for the individual $\mathrm{AUC}_{\mathrm{IR} 50}$ value calculated from the regression curve in this patient.

The curve between AUC and the growth IR approximated a logarithmic curve $\left(\mathrm{R}^{2}=0.655-0.999\right)$. The individual $\mathrm{AUC}_{\mathrm{IR} 50}$ value was calculated from the AUC-IR curve $\left(\mathrm{AUC}_{\mathrm{IR} 50}=\right.$ $8.1 \mu \mathrm{g}-98.8 \mathrm{~g} \mathrm{x} \mathrm{h} / \mathrm{ml}$ ) for all patients. The cumulative distribution of the individual $\mathrm{AUC}_{\mathrm{IR} 50}$ in 63 patients is shown in Fig. 2. The curve fits to two regression lines almost exactly
Table II. Backgrounds of the 45 resectable CRC patients.

\begin{tabular}{lccc}
\hline & Non-achieved & Achieved & \\
\hline No. of patients & 18 & 27 & NS \\
Mean age, years (range) & $65.9(35-78)$ & $65.0(30-79)$ & NS \\
Gender (male/female) & $8 / 10$ & $16 / 11$ & NS \\
Histological type & $1 / 15 / 0 / 2$ & $5 / 22 / 0 / 0$ & NS \\
(well/mod/poor/muc) & & & \\
Dukes' stage (A/B/C/D) & $0 / 8 / 10 / 0$ & $4 / 18 / 5 / 0$ & NS \\
Colon/rectum & $14 / 4$ & $23 / 4$ & NS \\
\hline
\end{tabular}

Well, well-differentiated; mod, moderately-differentiated; poor, poorly-differentiated; muc, mucinous carcinoma; NS, not significant.

Table III. Backgrounds of the 10 Dukes' D patients.

\begin{tabular}{lccc}
\hline & Non-achieved & Achieved & \\
\hline No. of patients & 5 & 5 & NS \\
Mean age, years (range) & $63.4(55-72)$ & $62.3(56-71)$ & NS \\
Gender (male/female) & $3 / 2$ & $3 / 2$ & NS \\
Histological type & $1 / 1 / 1 / 2$ & $0 / 4 / 0 / 1$ & NS \\
(well/mod/poor/muc) & & & \\
Colon/rectum & $4 / 1$ & $4 / 1$ & NS \\
\hline
\end{tabular}

Well, well-differentiated; mod, moderately-differentiated; poor, poorly-differentiated; muc, mucinous carcinoma; NS, not significant.

$\left[\mathrm{y}=0.0046 \operatorname{Ln}(\mathrm{x})+0.9154, \mathrm{R}^{2}=0.9657\right.$ and $\mathrm{y}=0.2268 \operatorname{Ln}(\mathrm{x})-$ $\left.0.7031, \mathrm{R}^{2}=0.9509\right]$. Patients on the regression line with a high $\mathrm{AUC}_{\mathrm{IR} 50}$ value accounted for $\sim 6 \%$ of all patients.

In all 63 patients, the relationship between the individual $\mathrm{AUC}_{\mathrm{IR} 50}$ and patient background, histological type (Fig. 3) and Dukes' stage (Fig. 4) was evaluated. No apparent trends were found for these parameters.

The backgrounds of the 45 resectable CRC patients according to group are shown in table II. In these patients, the background basis between the two groups was not significant. There was a trend towards a better DFS rate in the achieved group that failed to reach significance. The 5-year DFS rates were $88.9 \%$ for the achieved group and $72.2 \%$ for the non-achieved group (Fig. 5).

The backgrounds of the 10 Dukes' D patients according to group are shown in Table III. The background basis between the two groups was not significant. The OS rate of the achieved group was significantly better than that of the non-achieved group ( $\mathrm{P}=0.05$, Fig. 6$)$. The median survival time in the achieved group was 534 days (95\% CI 119-949), while in the non-achieved group it was 449 days (95\% CI 26-872). 


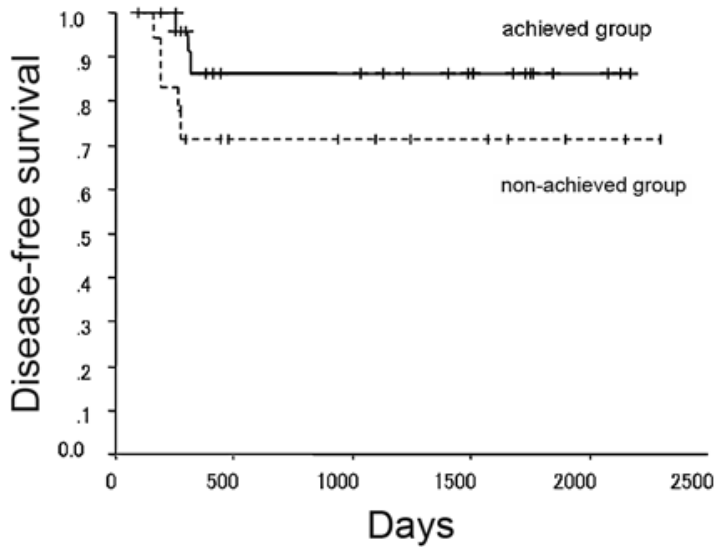

Figure 5. Disease-free survival rate of the achieved and non-achieved groups among the resectable colorectal cancer patients.

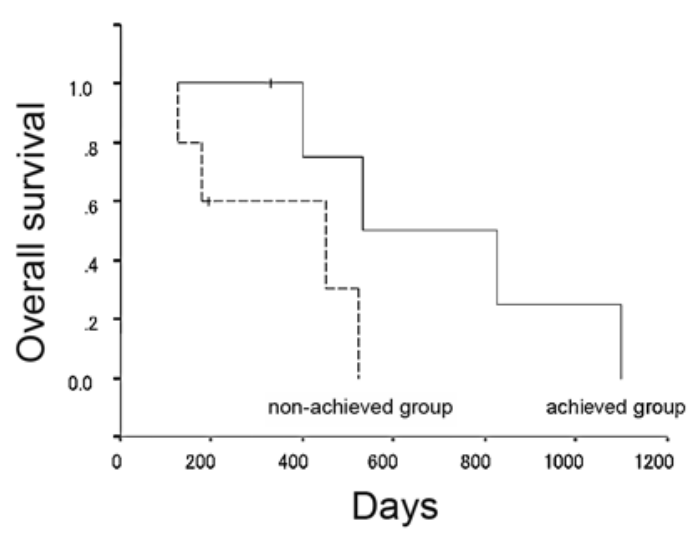

Figure 6. Overall survival of the achieved and non-achieved groups among the Dukes' D patients.

\section{Discussion}

Many meta-analyses show that there are responders and nonresponders in adjuvant chemotherapy for gastric cancer (4-8), though there is currently no useful method for distinguishing the two, as many steps are involved in the pharmacokinetics of anticancer drugs; e.g., absorption, metabolism and excretion. On the other hand, many in vivo factors affect the sensitivity of an anticancer drug; e.g., activation enzymes, degradation enzymes, membrane transport proteins, DNA repair enzymes and the target protein in the cancer cell. These factors are controlled by individual genes. Single nucleotide polymorphisms (SNPs), a type of gene polymorphism, are considered to be one of the factors affecting individual differences in the exertion of drug effects. Progress has recently been made in their analysis (9), but not enough for SNPs to be considered in individualized chemotherapy for intestinal cancer. Possible reasons for this are that the activity of the whole cancer type has been influenced by the living body, tumor and cell function, enzyme activity, the amounts of enzymes, mRNA expression and, lastly, gene polymorphisms. The cancer chemotherapy chemosensitivity test is therefore seen as very reasonable, and as the most useful in distinguishing responders and nonresponders. Iwahashi et al reported that overall survival was significantly better in a chemosensitivity-guided chemotherapy group than in a standard chemotherapy or non-chemotherapy group in advanced gastric cancer (10). There have been several studies employing the chemosensitivity assay in ovarian cancer, breast cancer and leukemia (11-14), while the number of studies using CD-DST in particular are increasing (15-24).

CD-DST is a method for the evaluation of drug sensitivity using isolated 3-dimensionally cultured tumor cells in a small collagen gel droplet (25). CD-DST has the following features: i) a high success rate due to the micro-3-dimensional culture; ii) the ability to work with a small quantity of specimen; iii) the ability to evaluate the anti-tumor effect of drugs in clinically equivalent doses; and iv) the ability to accurately evaluate the anti-cancer effect using an image analysis device when fibroblast contamination is $<67 \%$ (3). However, it is critical that the sample being examined be obtained from the soft part of the tumor tissue, in order to prevent the possible contamination of the fibroblast component.

In our previous report using CD-DST, the correlation between the 5-FU AUC and antitumor effect in colorectal cancer was evaluated, and in vitro AUC values and inhibition rates were found to be approximate to the logarithmic curve. Based on these results, we reported that the in vitro antitumor effect of 5-FU depends on the AUC in colorectal cancer, and that the AUC-IR curve is reliable. Moreover, we suggested that we were able to obtain the individual $\mathrm{AUC}_{\mathrm{IR} 50}$ - the AUC value that imparts 50\% growth inhibition - from the AUC-IR curve (2).

In this study, we calculated the individual $\mathrm{AUC}_{\mathrm{IR} 50}$ using the AUC-IR curve. Thus, 5-FU sensitivity is low in tumors with high $\mathrm{AUC}_{\mathrm{IR} 50}$ values. The cumulative distribution of the individual $\mathrm{AUC}_{\mathrm{IR} 50}$ curve fitted to two regression lines. Patients on the high $\mathrm{AUC}_{\mathrm{IR} 50}$ regression line were poor responders in vitro, while patients on the other regression line were goodto-intermediate responders in vitro. The first step for individualized 5-FU-based chemotherapy is the selection of these poor responders, for whom an alternative regimen is appropriate. Favourable responders are candidates for shorter-term standard 5-FU-based chemotherapy; e.g., oral 5-FU, oxaliplatin plus 5 -FU or leucovorin (FOLFOX)/irinotecan plus 5-FU and leucovorin (FOLFIRI), whereas intermediate responders are candidates for longer-term or more intensive 5-FU-based chemotherapy; e.g., FOLFOX with molecular target drugs, such as bevacizumab and cetuximab/FOLFIRI with molecular target drugs. However, in chemotherapy for poor responders, 5-FU is always included in the standard regimens, and the single response rates of irinotecan and oxaliplatin are not as high. In individualized chemotherapy against colorectal cancer, this problem is in need of further study.

Tumor histological type analysis showed no significant correlation with $\mathrm{AUC}_{\mathrm{IR} 50}$. In other words, poorly-differentiated adenocarcinoma and mucinous carcinoma are not necessarily poor responders in vitro. Therefore, we should not discount 5-FU-based chemotherapy for poorly-differentiated adenocarcinoma and mucinous carcinoma. However, Dukes' stage analysis showed no significant correlation with the individual $\mathrm{AUC}_{\mathrm{IR} 50}$. The progress of Dukes' stage does not always indicate 5-FU refractory cancer, and Dukes' $\mathrm{D}$ patients are not necessarily poor responders in vitro. Therefore, 5-FU-based chemotherapy should also not be discounted for Dukes' D patients. 
Analysis of the resectable CRC patients treated with 5-FUbased adjuvant chemotherapy showed that the recurrence rate of the non-achieved group (total administered AUC less than individual $\mathrm{AUC}_{\mathrm{IR} 50}$ ) was higher than that of the achieved group (total administered AUC greater than individual $\mathrm{AUC}_{\mathrm{IR} 50}$ ). Moreover, the DFS rate of the achieved group was better than that of the non-achieved group. Therefore, this study suggests that the achievement of the individual $\mathrm{AUC}_{\mathrm{IR} 50}$ is a prerequisite for individualized 5-FU-based adjuvant chemotherapy. In contrast, analysis of Dukes' D patients treated with 5-FU-based chemotherapy revealed that the OS rate of the achieved group (total administered AUC more than twice the individual $\mathrm{AUC}_{\mathrm{IR} 50}$ ) was significantly better than that of the non-achieved group (total administered AUC less than twice the individual $\left.\mathrm{AUC}_{\mathrm{IR} 50}\right)$. These findings indicate that the early achievement of twice the individual $\mathrm{AUC}_{\mathrm{IR} 50}$ is associated with improved prognosis in Dukes' D patients.

In the present study, we were able to obtain the individual $\mathrm{AUC}_{\mathrm{IR} 50}$ from the AUC-IR curve using CD-DST, and to select poor responders in the cumulative distribution of the individual $\mathrm{AUC}_{\mathrm{IR} 50}$ curve. The results also suggest that individual $\mathrm{AUC}_{\mathrm{IR} 50}$ is useful in the establishment of individualized chemotherapy for colorectal cancer patients.

\section{References}

1. Ochiai T, Nishimura K, Noguchi H, Kitajima M, Tsuruoka Y and Takahashi Y: Evaluation of 5-fluorouracil applicability by multi-point collagen gel droplet embedded drug sensitivity test Oncol Rep 14: 201-205, 2005.

2. Ochiai T, Nishimura K, Noguchi H, et al: Evaluation of 5-fluorouracil applicability by the collagen gel droplet embedded drug sensitivity test with area under the curve analysis. Anticancer Drugs 18: 17-21, 2007.

3. Kobayashi H, Higashiyama M, Minamigawa K, et al: Examination of in vitro chemosensitivity test using collagen gel droplet culture method with colorimetric endpoint quantification. Jpn J Cancer Res 92: 203-210, 2001.

4. Hermans J, Bonenkamp JJ, Boon MC, et al: Adjuvant therapy after curative resection for gastric cancer: meta-analysis of randomized trials. J Clin Oncol 11: 1441-1447, 1993.

5. Earle CC and Maroun JA : Adjuvant chemotherapy after curative resection for gastric cancer in non-Asian patients: revisiting a meta-analysis of randomised trials. Eur J Cancer 35: 1059-1064, 1999.

6. Mari E, Floriani I, Tinazzi A, et al: Efficacy of adjuvant chemotherapy after curative resection for gastric cancer: a meta-analysis of published randomised trials. A study of the GISCAD (Gruppo Italiano per lo Studio dei Carcinomi dell'Apparato Digerente). Ann Oncol 11: 837-843, 2000.

7. Janunger KG, Hafstrom L, Nygren P, Glimelius B; SBU-group, Swedish Council of Technology Assessment in Health Care: A systematic overview of chemotherapy effects in gastric cancer. Acta Oncol 40: 309-326, 2001.

8. Panzini I, Gianni L, Fattori PP, et al: Adjuvant chemotherapy in gastric cancer: a meta-analysis of randomized trials and a comparison with previous meta-analyses. Tumori 88: 21-27, 2002.
9. Kitajima M, Takita N, Hata M, et al: The relationship between 5-fluorouracil sensitivity and single nucleotide polymorphisms of the orotate phosphoribosyl transferase gene in colorectal cancer. Oncol Rep 15: 161-165, 2006.

10. Iwahashi M, Nakamori M, Nakamura M, et al: Individualized adjuvant chemotherapy guided by chemosensitivity test sequential to extended surgery for advanced gastric cancer. Anticancer Res 25: 3453-3459, 2005.

11. Cree IA, Kurbacher CM, Untch M, et al: Correlation of the clinical response to chemotherapy in breast cancer with ex vivo chemosensitivity. Anticancer Drugs 7: 630-635, 1996.

12. Kurbacher CM, Bruckner HW, Cree IA, et al: Mitoxantrone combined with paclitaxel as salvage therapy for platinumrefractory ovarian cancer; laboratory study and clinical pilot trial. Clin Cancer Res 3: 1527-1533, 1997.

13. Andreotti PE, Cree IA, Kurbacher CM, et al: Chemosensitivity testing of human tumors using a microplate adenosine triphosphate luminescence assay: clinical correlation for cisplatin resistance of ovarian carcinoma. Cancer Res 55: 5276-5282, 1995.

14. Staib P, Staltmeier E, Ncurohr K, Cornely O, Reiser M and Schinkothe T: Prediction of individual response to chemotherapy in patients with acute myeloid leukaemia using the chemosensitivity index Ci. Br J Haematol 128: 783-791, 2005.

15. Takamura Y, Kobayashi H, Taguchi T, Motomura K, Inaji $\mathrm{H}$ and Noguchi S: Prediction of chemotherapeutic response by collagen gel droplet embedded culture-drug sensitivity test in human breast cancers. Int J Cancer 98: 450-455, 2002.

16. Fujii R, Seshimo A and Kameoka S: Relationships between the expression of thymidylate synthase, dihydropyrimidine dehydrogenase, and orotate phosphoribosyltransferase and cell proliferative activity and 5-fluorouracil sensitivity in colorectal carcinoma. Int J Clin Oncol 8: 72-78, 2003.

17. Mori S, Kunieda K, Sugiyama Y and Saji S: Prediction of 5-fluorouracil and cisplatin synergism for advanced gastrointestinal cancers using a collagen gel droplet embedded culture. Surg Today 33: 577-583, 2003.

18. Yabushita H, Ohnishi M, Komiyama M, et al: Usefulness of collagen gel droplet embedded culture drug sensitivity testing in ovarian cancer. Oncol Rep 12: 307-311, 2004.

19. Inoue Y, Gika M, Abiko T, et al: Bcl-2 overexpression enhances in vitro sensitivity against docetaxel in non-small cell lung cancer. Oncol Rep 13: 259-264, 2005.

20. Kawaguchi M, Banno K, Susumu N, et al: Successful analysis of anticancer drug sensitivity by CD-DST using pleural fluid and ascites from patients with advanced ovarian cancer: case reports. Anticancer Res 25: 3547-3551, 2005.

21. Okumura K, Shiomi H, Mekata E, et al: Correlation between chemosensitivity and mRNA expression level of 5-fluorouracilrelated metabolic enzymes during liver metastasis of colorectal cancer. Oncol Rep 15: 875-882, 2006.

22. Kawamura M, Gika M, Abiko T, et al: Clinical evaluation of chemosensitivity testing for patients with unresectable nonsmall cell lung cancer (NSCLC) using collagen gel droplet embedded culture drug sensitivity test (CD-DST). Cancer Chemother Pharmacol 59: 507-513, 2007.

23. Shimizu T, Murata S, Mekata E, et al: Clinical potential of an antitumor drug sensitivity test and diffusion-weighted MRI in a patient with a recurrent solid pseudopapillary tumor of the pancreas. J Gastroenterol 42: 918-922, 2007.

24. Okumura K, Mekata E, Shiomi H, et al: Expression level of thymidylate synthase mRNA reflects 5-fluorouracil sensitivity with low dose and long duration in primary colorectal cancer. Cancer Chemother Pharmacol 61: 587-594, 2008.

25. Matsuo A, Watanabe A, Takahashi T, et al: A simple method for classification of cell death by use of thin layer collagen gel for the detection of apoptosis and/or necrosis after cancer chemotherapy. Jpn J Cancer Res 92: 813-819, 2001. 